\title{
Influence of Climate on Pneumocystosis Cases in the Eastern Brazilian Amazon
}

Camilla da Silva Machado ${ }^{1}$, Victor Ferreira Abreu ${ }^{1}$, Pedro Henrique Cunha Fontenelle ${ }^{1}$, Alessandra Teixeira de Macedo ${ }^{1}$, Haryne Lizandrey Azevedo Furtado ${ }^{1}$, Conceição de Maria Pedrozo e Silva de Azevedo², Rodrigo Assuncao Holanda ${ }^{3}$ and Julliana Ribeiro Alves Santos ${ }^{1}$

1. Laboratório de Microbiologia Ambiental, Universidade CEUMA, RuaJosué Montello, 01, São Luís, Maranhão 65075-120, Brazil

2. Centro de Ciências da Saúde, Universidade Federal do Maranhão, Av. dos Portugueses, 1966-Vila Bacanga, São Luís, MA 65080-805, Brazil

3. Laboratório de Biologia Molecular de Microrganismos Patogênicos, Universidade CEUMA, RuaJosué Montello, 01, São Luís, Maranhão 65075-120, Brazil

\begin{abstract}
Here, we evaluated the clinical aspects and environmental factors for five cases of pneumocystosis in the eastern Brazilian Amazon. Pneumocystosis was associated with human immunodeficiency virus (HIV) infection, male sex, age (until 30 years), and treatment with sulfamethoxazole plus trimethoprim in $100 \%, 80 \%, 40 \%$, and $80 \%$ cases, respectively. Drier months, higher temperatures, and wind speed were favorable variables for pneumocystosis. Overall, this fungal infection may be related to global warming, resulting in an increased occurrence of cases.
\end{abstract}

Key words: Pneumocystosis, HIV, climate, temperature, Amazon.

\section{Introduction}

Pneumocystis jirovecii is a single-celled eukaryotic organism found in different environments. It specifically infects humans with tropism in the upper respiratory tract, which can cause severe interstitial pneumonia known as pneumocystosis [1]. The transmission of $P$. jirovecii has not been fully elucidated yet and its ecological niche remains unknown; however, the development of this infection is known to depend on the host immunological condition. For this reason, pneumocystosis is found more frequently in immune suppressed patients, especially in those with acquired immunodeficiency syndromeand in those infected with human immunodeficiency virus (HIV) [2], and has been known to increase in cases of patients with

Corresponding author: Julliana Ribeiro Alves Santos, $\mathrm{PhD}$, research fields: health and environment, medical mycology, antimicrobial resistance, pathogen-host interaction. hematological malignancies, inflammatory and autoimmune diseases, and multi-organ tumors [3].

Since the discovery of the association between $P$. jirovecii and HIV infections, pneumocystosis has gained increasing importance; with an estimated $80 \%$ of HIV carriers developing pneumonia, pneumocystosis has been considered one of the main causes of mortality in the United States of America [4].

Due to the constant silent evolution of HIV-positive patients and symptoms that can be confused with other diseases already present in the host, accurate diagnosis of pneumocystosis is often difficult [5]. Therefore, pneumocystosis treatment is often performed in the final stages of the disease and many HIV-positive patients die before undergoing treatment for pneumocystosis. The most commonly used drugs for treating this disease include trimethoprim-sulfamethoxazole, pentamidine, dapsone, and atovaquone [5].

Recently, Silva et al. [6] described the influence of 
climate on mycoses hospitalizations in the capitals of Brazil; in addition, Silva et al. [7] showed that mycoses are an important indicator of socio environmental vulnerability in the same Brazilian Amazon-Savanna transition region described in this study.

In the present case series, we describe the environmental influence in five cases of pneumocystosis in the Brazilian Amazon-Savanna transition region in the state of Maranhão, Brazil.

\section{Case Series}

The data onpatients' sex, age, length of stay, city, and examinations performed, diagnosis, treatment, and outcomes were obtained. In total, five patients were admitted to the Hospital Unit, one each in October 2018, November 2018, and in May, July, and August 2019.

There were four males and one female, between the

A

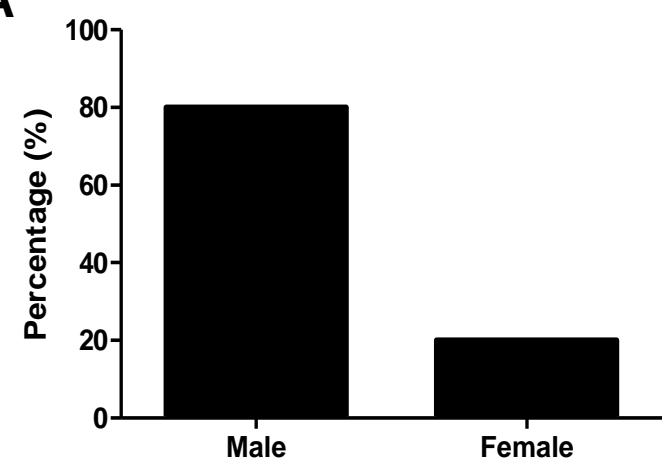

C

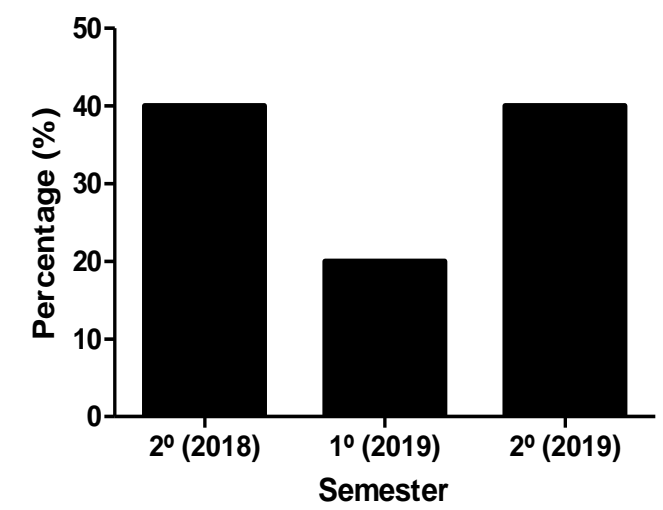

ages of 25 to 30 years $(n=2), 35$ to 40 years $(n=1)$, 41 to 46 years $(n=1)$, and 47 to 51 years $(n=1)$. The occurrence of pneumocystosis was higher in the second semester of 2018 and in the second semester of 2019. The city with the most cases of infection was São Luís, the capital of Maranhão, representing four cases (Fig. 1).

All the patients underwent blood tests as well as immunological and biochemical tests (Fig. 2a). Based on the test results, all patients were diagnosed with pneumocystosis associated with HIV infection (Fig. 2b). For treatment, the most used drugs were the combinations of sulfamethoxazole with trimethoprim (80\%), followed by fluconazole, metronidazole, and azithromycin (Fig. 2c). Of all the patients, $40 \%$ were discharged, $40 \%$ died, and 20\% remained hospitalized until the end of this study (Fig. 2d).

B

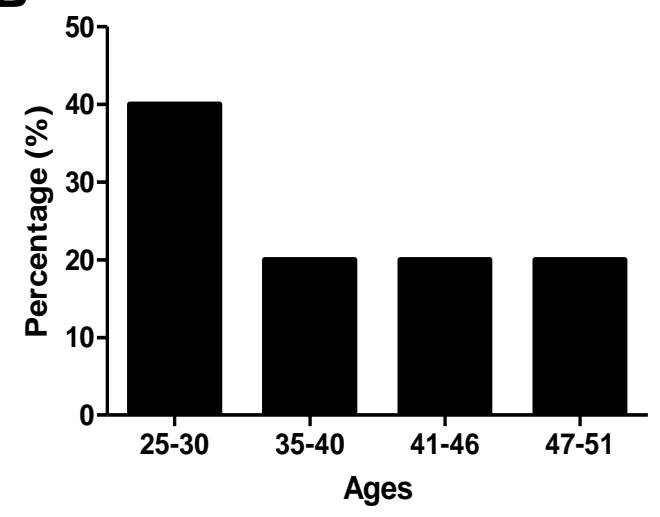

D

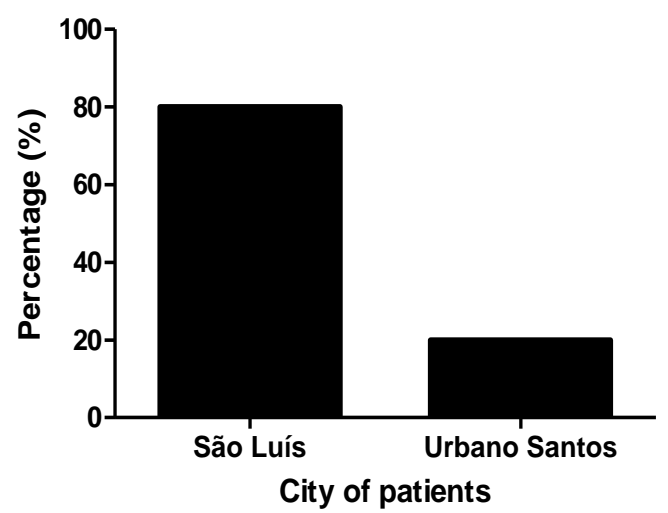

Fig. 1 (a)Percentage of pneumocystosis cases associated with sex, (b)percentage of cases associated with age, (c)percentage of cases associated with the semester of each year, (d)percentage of cases associated with city of patients. 
A

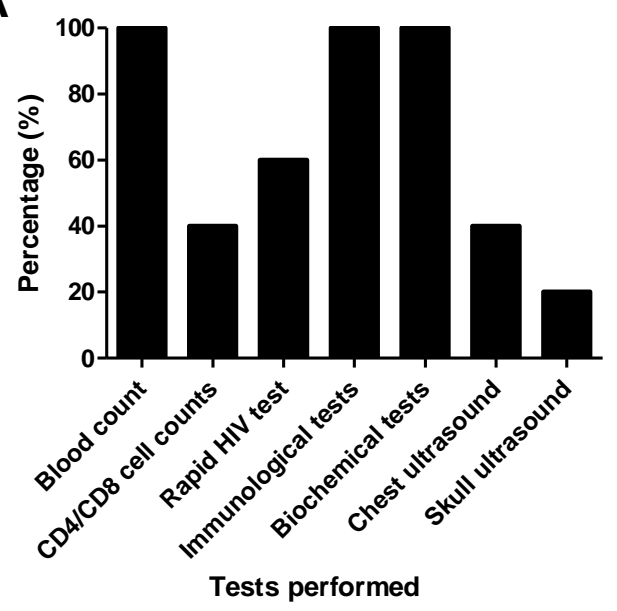

C

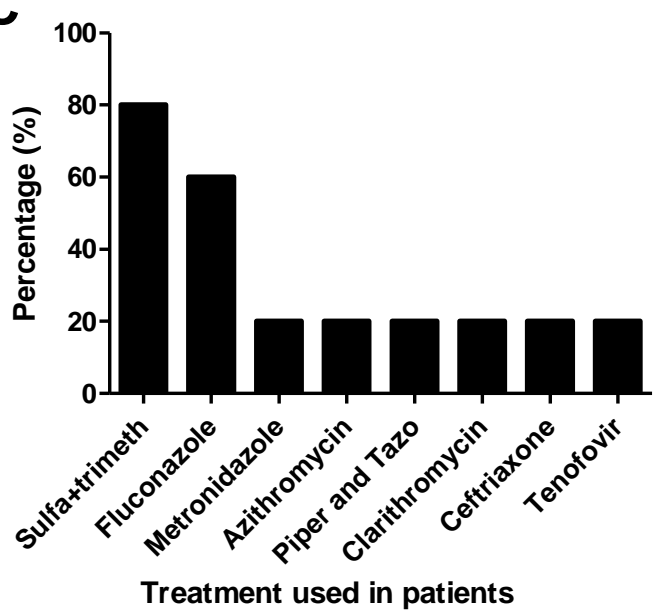

B

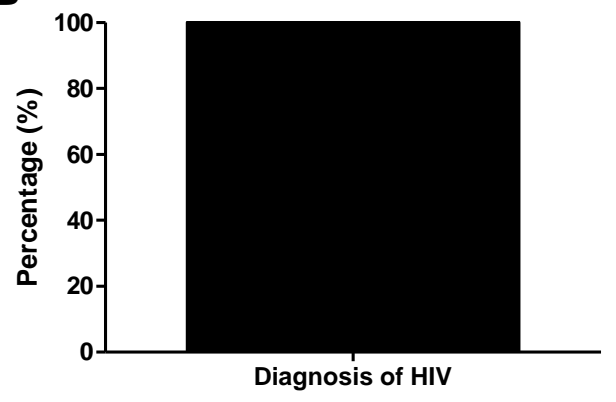

D

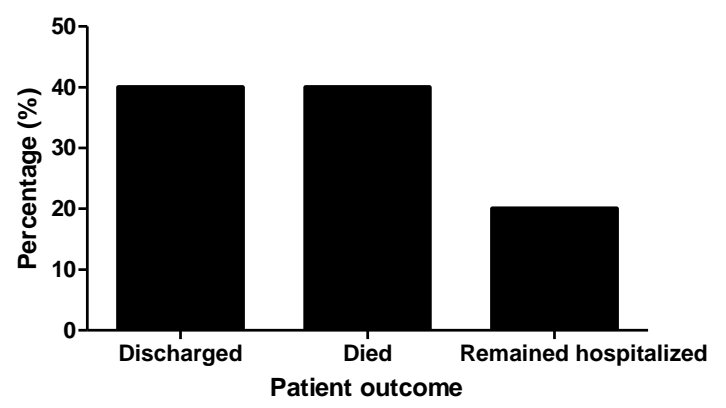

Fig. 2 Laboratory, clinical, and treatment aspects of patients: (a) tests performed, (b) diagnosis of HIV-associated pneumocystosis, (c) treatment used in patients, and (d) patient outcome. Legend: Sulfa + trimeth: sulfamethoxazole + trimethoprim. Piper and Tazo:piperacillin and tazobactam.

A temporal analysis of pneumocystosis cases and the social and environmental aspects was carried out. The environmental aspects chosen were total precipitation, maximum, minimum and average temperatures, and average wind speed. In the months of October and November 2018, and May, July, and August 2019, when patients were hospitalized, the total precipitation was lower (Fig. 3a), whereas the maximum, minimum, and average temperatures (Fig. $3 \mathrm{~b})$, and the average wind speed were higher compared to those in the other months (Fig. 3c) in the capital, São Luís (Fig. 3d). In Urbano Santos, lower rates of total precipitation were observed in October and November 2018, and in July and August 2019,
(Fig. 3a). However, the month of May showed higher rates when compared to other months. The maximum and average temperatures (Fig. 3b), as in São Luís, were high, whereas the minimum temperature in the months of May and July was lower when compared to the other months.

The study site is in a transition region between two important Brazilian biomes, the Amazon and the Savanna. These have differences between their precipitation and temperature levels, which may influence cases of mycoses such as pneumocystosis.

In compliance with the requirements demanded by Resolution 466/12 from the National Health Council for research involving human beings, the Research 

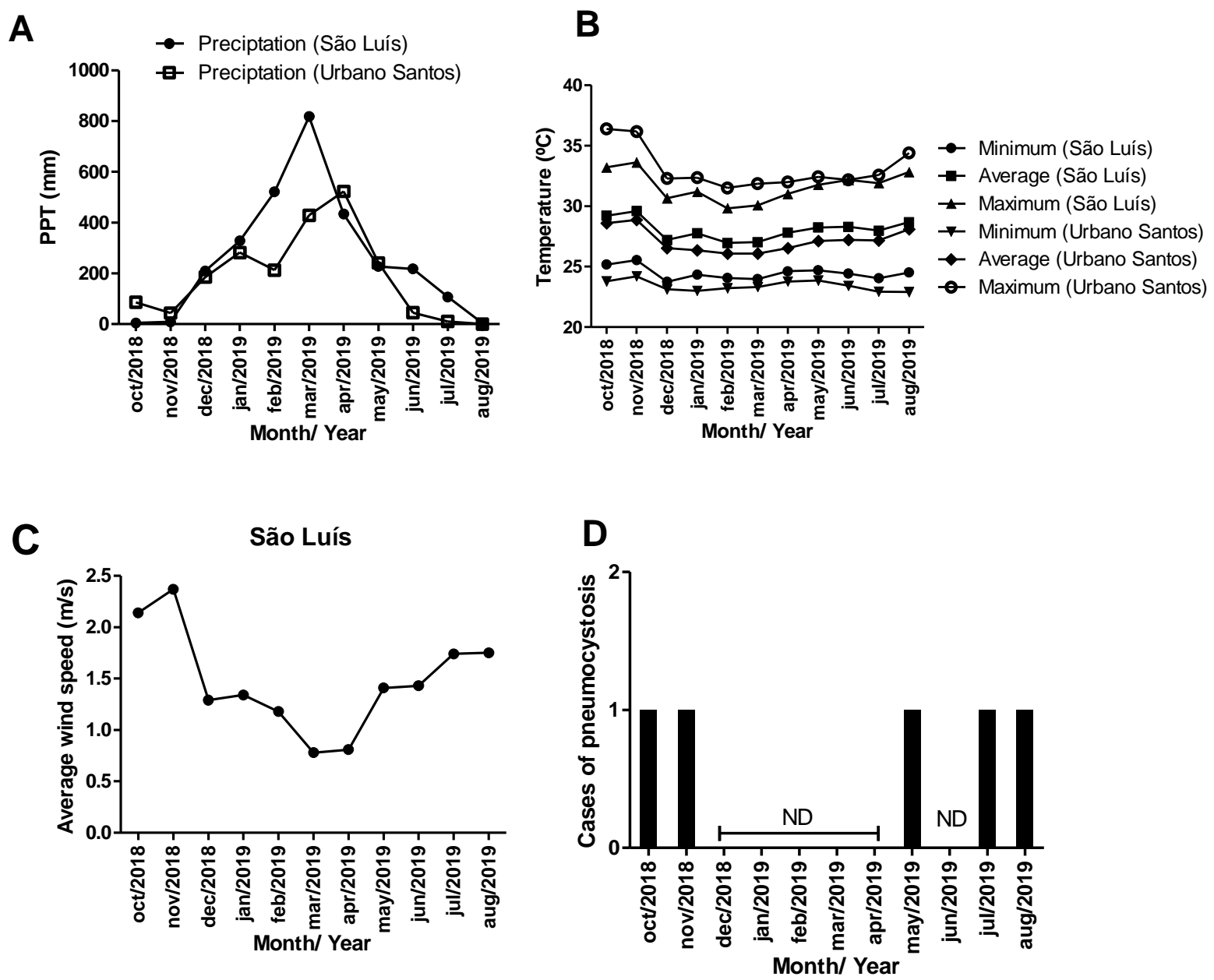

Fig. 3 Climatic conditions in the municipality of São Luís and Urbano Santos: (a) total precipitation, (b) minimum, average, and maximum temperatures, (c) average wind speed in São Luís, (d) time series of cases of pneumocystosis. ND: non-detected.

Project was approved by the Research Ethics Committee of Universidade Ceuma, under number $2,927,864$.

\section{Discussion}

The present study was the first to evaluate the cases of pneumocystosis in the transition region between the Amazon and Savanna, considering the environmental aspects. Thus, the number of cases in the second semester of 2018 and 2019 was possibly related to the environmental conditions during these months of the study.

According to Melchior et al. [8], the most requested examinations for investigating suspected pathologies in patients with HIV infection are simple laboratory tests such as chest and abdomen radiography (90\%), whereas imaging tests showed lower availability (75.2\%). The authors also reported that CD4/CD8 counts and viral load are available in most services offered in the Unified Health System, but are not performed (41\% do not perform CD4/CD8 counts and $59 \%$ do not perform viral load).

The analysis by Melchior et al. [8] and Junior et al. [9] was similar to the present study, but the amount of rapid tests performed was higher than the CD4/CD8 cell counts performed and the bronchoscopy exam with bronchoalveolar lavage was not performed for the patients evaluated, which can be explained by the 
greater availability of rapid tests in the study reference hospital in relation to the CD4/CD8 count, and a lower availability of bronchoscopy exams with bronchoalveolar lavage.

The combination of sulfamethoxazole and trimethoprim was the most used treatment. In addition to antifungal drugs, it is important to use antiretroviral drugs to treat HIV infection for improving the outcomes of pneumocystosis treatment.

The present study reports parity between the cases of death and discharge in patients who presented pneumocystosis, which can be caused by the severe conditions in these patients. According to Ribeiro [5], about $58.8 \%$ of patients who were diagnosed with HIV-associated pneumocystosis died, whereas $41.2 \%$ were discharged. According to Jain et al. [10], despite a decrease in the cases of death in patients with HIV after the emergence of potent antiretroviral therapy, pneumocystosis persists as one of the main causes of death in these individuals.

In a study carried out by Miller et al. [11] in London, four genotypes of $P$. jirovecii were observed. The first genotype was found in greater quantities in the month of May, when rainfall was lower $(40 \mathrm{~mm})$ and the temperature was higher $\left(15^{\circ} \mathrm{C}\right)$ compared to other months such as February, January, and April.

Mycoses hospitalizations are also influenced by the climate in different capitals of Brazil [6]. According to Silva et al. [6], the minimum temperature is the key climatic variable for the pattern of hospitalizations due to mycoses. In the city of Urbano Santos, the minimum temperature was observed to be lower in May and July 2019, in which cases of pneumocystosis were not observed; this could explain the disagreement in relation to the other months.

A positive correlation has been observed between the incidence of pneumocystosis and climatic factors such as average temperature [12], and climatological and environmental air pollution factors [13, 14]. In San Francisco (USA), changes in climatic factors and high levels of the polluting constituents in ambient air, temperature, and $\mathrm{SO}_{2}$ were observed immediately (0-3 days) before pneumocystosis-related admission among HIV-positive patients living in the city [13].

\section{Conclusions}

Pneumocystosis is a disease caused by a fungus, whose mechanisms of infection and development in the host body remain unknown. However, the cases of pneumocystosis are increasing worldwide, due to a lack of information and delayed and flawed diagnoses.

Considering this, studies related to pneumocystosis should be encouraged so that the number of cases in Brazil and worldwide, can be decreased.

\section{Acknowledgements}

We would like to thank the Universidade CEUMA (UNICEUMA, São Luis-MA, Brazil) and Fundação de Amparo a Pesquisa e Desenvolvimento do Estado do Maranhão-FAPEMA (Grant UNIVERSAL-01164/17). JRAS is a research fellow of the FAPEMA (Grant BEPP-02494/18).

\section{Conflicts of Interest}

The authors declare no conflicts of interest.

\section{References}

[1] Esteves, F., Antunes, F., and Matos, O. 2014. "Pneumocystis and Pneumocystosis: The Pathogen and the Disease." Rev Port de Doenças Infecciosas 10 (1): 16-22.

[2] Tomio, D., and Da Silva, R. M. 2005. "Pneumocistose." Arq Catarinenses Med 34 (4): 85-91.

[3] Lee, H.-Y., Choi, S.-H., Kim, T., Chang, J., Kim, S.-H., Lee, S. O., et al. 2019. "Epidemiologic Trends and Clinical Features of Pneumocystis jirovecii Pneumonia in Non-HIV Patients in a Tertiary-Care Hospital in Korea over a 15-Year-Period." Japan J Infect Dis 2 (4): 270-3.

[4] Giehl P. A. S. M., Sanches, F. G., Ribeiro, P. C., Carvalho, R. W., Serra-Freire, N. M., and Norberg, A. N. 2014. "Pneumocystis Associated to the Human Immunodeficiency Virus in the City of Rio de Janeiro, Brazil." Rev Cubana Med Trop 66 (1).

[5] Ribeiro, L. C., Hahn, R. C., Favalessa, O. C., Tadano, T., and Fontes, C. J. F. 2009. "Systemic Mycosis: Factors Associated with Death among Patients Infected with the 
Human Immunodeficiency Virus, Cuiabá, State of Mato Grosso, Brazil, 2005-2008." Rev Soc Bras Med Trop 42 (6): 698-705.

[6] Silva, F. B., Santos, J. R. N., Silva, L. C., Gomes, W. C., Villis, P. C. M., Gomes, E. S. et al. 2019. "Climate Drivers of Hospitalizations for Mycoses in Brazil." Sci Rep 9: 6902.

[7] Silva, L. C., Oliveira, L. V. N., Silva, F. B., Santos, J. R. N., Araújo, M. L. S., Mendes, A. G. G. et al. 2020. "Hospitalisations for Mycoses as an Indicator of Socio-Environmental Vulnerability in the Brazilian Amazon-Savanna Transition Region." Mycoses 63: 151-61.

[8] Melchior, R., Nemes, M. I. B., Basso, C. R., Castanheira, E. R. L., De Britto e Alves, M. T. S., Buchalla, C. M. et al. 2006. "Evaluation of the Organizational Structure of HIV/AIDS Outpatient Care in Brazil." Rev Saúde Públ 40: 143-51.

[9] Junior, L. G. C., Souza, D. L., Machado, G. B., Silva, J. S., and Monteiro, M. L. G. R. 2016. "Pneumocystosis Diagnosed during Pregnancy: Case Report." Rev Med 95 (3): $146-51$
[10] Jain, M. K., Skiest, D. J., Cloud, J. W., Jain, C. L., Burns, D., and Berggren, R. E. 2003. "Changes in Mortality Related to Human Immunodeficiency Virus Infection: Comparative Analysis of Inpatient Deaths in 1995 and in 1999-2000." Clin Infect Dis 36 (8): 1030-8.

[11] Miller, R. F., Evans, H. E. R., Copas, A. J. and Cassell, J. A. 2007. "Climate and Genotypes of Pneumocystis jirovecii." Clin Microbiol Infect 13 (4): 445-8.

[12] Sing, A., Schmoldt, S., Laubender, R. P., Heesemann, J., Sing, D. and Wildner, M. 2009. "Seasonal Variation of Pneumocystis jirovecii Infection: Analysis of Underlying Climatic Factors." Clin Microbiol Infect 15 (10): 957-60.

[13] Djawe, K., Levin, L., Swartzman, A., Fong, S., Roth, B., Subramanian, A.,et al. 2013. "Environmental Risk Factors for Pneumocystis Pneumonia Hospitalizations in HIV Patients." Clin Infect Dis 56 (1):74-81.

[14] Miller, R. F., Huang, L. and Walzer, P. D. 2018. "The Relationship between Pneumocystis Infection in Animal and Human Hosts, and Climatological and Environmental Air Pollution Factors: A Systematic Review." OBM Genet 2 (4). 